\title{
Prognostic Factors for Successful Percutaneous Disc Decompression Using the Navigable Device L'DISQ in Patients with Lumbar Discogenic Pain
}

\section{Ji Yeong Kim}

Yonsei University College of Medicine

Ku Sang Lee

The Catholic University of Korea

Sang Min Jung

The Catholic University of Korea

Young Hoon Kim ( $\square$ anekyh@catholic.ac.kr)

The Catholic University of Korea

\section{Research Article}

Keywords: percutaneous disc decompression (PDD), device L'DISQ, lumbar discogenic pain

Posted Date: July 9th, 2021

DOI: https://doi.org/10.21203/rs.3.rs-692009/v1

License: (c) (i) This work is licensed under a Creative Commons Attribution 4.0 International License. Read Full License 


\section{Abstract}

The navigable percutaneous disc decompression (PDD) device L'DISQ is an effective and safe option for the treatment of lumbar discogenic pain. This retrospective study aimed to evaluate the prognostic factors associated with the successful outcome of PDD using the L'DISQ for treating lumbar discogenic pain by following up patients before and 1, 2, 3, and 6 months after the procedure. A successful outcome was defined as $a \geq 50 \%$ reduction in the numeric rating scale scores for pain and $a \geq 40 \%$ reduction in the Oswestry disability index scores at 6 months after the procedure. Clinical parameters and patient demographics, including pain duration, history of surgery, number of treatment levels, and radiographic findings of lumbar magnetic resonance imaging, were also examined. Of the 106 patients included, 80 (75.5\%) had successful outcomes at 6 months. Multivariable logistic regression analysis revealed that the presence of high-intensity zones $(\mathrm{HIZs})(\mathrm{P}=0.016)$ was an independent positive predictor of successful PDD outcomes; conversely, migration of the herniated disc $(P=0.017)$ and bilaterally herniated discs $(P=0.001)$ were negative predictors. Therefore, the presence of HIZs, absence of migration of herniated discs, and presence of unilaterally herniated discs are positive predictors of successful outcomes.

\section{Introduction}

Lumbar discogenic pain accounts for $40 \%$ cases of chronic lower back pain ${ }^{1}$. The most common pathophysiology of this pain is annular disruption ${ }^{2}$. When posterior annular damage causes the nucleus pulposus to migrate to the outer annulus, the protruded disc stimulates the nociceptors in the posterior annulus and posterior longitudinal ligament of the dural sac ${ }^{3,4}$. Inflammatory cytokines produced by ingrowing nerve endings also stimulate nociceptors. These chemical and mechanical processes contribute to the development of lumbar discogenic pain ${ }^{5-7}$. Although no standardised treatment for lumbar discogenic pain is currently available, open disc surgery could be considered if conservative treatments, such as medication or physiotherapy, are ineffective ${ }^{8-11}$. However, open disc surgery is invasive and can cause severe complications in some cases ${ }^{12,13}$. As an alternative, minimally invasive disc decompression has been used over the past several decades. Compared to open surgery, this procedure reduces pain severity while maintaining the normal disc tissue ${ }^{14-17}$. Moreover, it can be performed using various techniques, such as intradiscal electrothermal therapy, intradiscal injection, and nucleoplasty ${ }^{18-23}$.

Partial disc decompression is certainly safer than open surgery, but most studies have shown that it has a lower success rate than open and micro-discectomy in reducing radicular pain ${ }^{18,24,25}$. Introduced in 1999, nucleoplasty (Arthrocare Co., Sunnyvale, CA) is a representative nuclear decompression technique that removes nuclear tissue using bipolar radiofrequency energy -the so-called coblation technologyapplied to a saline-conducting medium ${ }^{24,26}$. However, like most decompression techniques, this is also limited by the difficulty in accessing the outer annulus. Moreover, nuclear decompression via nucleoplasty mainly relies on lowering the pressure rather than directly removing the extruded disc. 
The navigable percutaneous disc decompression (PDD) device L'DISQ (U\&I Co., Uijeongbu, Korea) was designed to direct herniated disc material through a de novo wand that can be curved by rotating a control wheel. This device ablates the herniated nucleus by using a bipolar radiofrequency energy similar to that used in nucleoplasty. It also coagulates the sinuvertebral nerve that innervates the intervertebral disc. Several previous studies have reported the clinical effects of PDD using the L'DISQ in lumbar discogenic pain, but none have yet reported the prognostic factors of the procedure ${ }^{27,28}$. Herein, we evaluated various factors associated with the efficacy of PDD using the L'DISQ for managing lumbar discogenic pain.

\section{Methods}

\section{Patients.}

We reviewed the medical records of patients who underwent PDD using L'DISQ following a diagnosis of lumbar discogenic pain between January 2015 and July 2020 at a tertiary university hospital. All procedures were performed by a single physician (Y.H.K.). The study was approved and informed consent was waived by The Catholic University of Korea, Seoul St. Mary's Hospital Institutional Review Board (IRB No. KC21IRSI0101). This retrospective cohort study was registered with CRIS (Clinical Research Information Service of the Korea National Institute of Health, https://cris.nih.go.kr/cris/index.jsp, KCT0005967). All methods were performed in accordance with the relevant guidelines and regulations. All medical data were collected by a standardized protocol, and only analysed after anonymisation. The inclusion criteria were as follows: (1) age at least 20 years old; (2) numeric rating scale for pain (NRS pain) score of 5 or more; (3) diagnosis of lumbar intervertebral disc herniation confirmed using magnetic resonance imaging (MRI) and patient-reported concordant back pain or radicular pain in the lower extremity, or both; (4) pain duration of 3 months or more; (5) failure to respond to previous epidural injections combined with exercise and pharmacotherapy. The exclusion criteria were as follows: (1) absence of lumbar spine MRI; (2) poorly controlled coexisting psychiatric diagnosis; (3) postherpetic neuralgia in the lumbar spinal nerve, or any other condition potentially accounting for the signs and symptoms; (4) lumbar myelopathy or any evidence of central nervous system injury; (5) presence of coagulopathy, malignancy, fever, or local infection at the puncture site.

\section{PDD using the L'DISQ.}

Intravenous access was prepared in advance, and prophylactic antibiotics were injected 30 min before the start of the procedure. The vital signs of the patient, including pulse oximetry, electrocardiogram, and blood pressure, were monitored continuously during the procedure. The patient was kept in a prone position, and a pillow was placed under the abdomen to minimise lumbar lordosis. The skin was disinfected with povidone-iodine and draped in a sterile manner. The endplate of the target disc was aligned and the $\mathrm{C}$-arm was then rotated until the lateral margin of the contralateral superior articular process passed the posterior 3/5th of the vertebral body. Local anaesthesia was administered in this 
view, while ensuring the needle entry site was $12-15 \mathrm{~cm}$ from the midline. The introducer needle was directed toward the lateral edge of the contralateral superior articular process, where it would meet the disc margin at the line drawn between the medial borders of the adjacent pedicles in the anteroposterior view. When the needle passed through the annulus fibrosus, the physician felt a sudden loss of resistance. When the needle reached the border of the annulus fibrosus and nucleus pulposus, it stopped advancing. The stylet was then removed, and only the introducer was retained. The wand of the L'DISQ was placed into the introducer. Depending on the position of the target disc, the lever was pulled and the tip of the wand was adjusted to finally reach the target area of the lesion. After reaching the target area, a low-voltage (0.5-1 V) electrical stimulation was applied. If the patient experienced a momentary, sharp, or sudden aching sensation in the back or the buttock, the tip of the wand was repositioned. The ablation and coagulation modes were used as per established protocols ${ }^{27,28}$. All adverse events that occurred during and after the procedure were recorded. After the procedure, the patient was transferred to the recovery room, and vital signs were monitored. After a few hours of recovery, the patient was discharged once a neurological examination confirmed the absence of any abnormalities.

\section{Data collection.}

Patient demographic and clinical data, including age, sex, height, weight, smoking history, pain duration, and history of surgery, were recorded. The NRS pain scores before and at 1,2,3, and 6 months after the procedure were assessed. The functional status was estimated using the Oswestry disability index (ODI) before and at 1, 2, 3, and 6 months after the procedure. The findings of radiographic examinations, including plain radiography and lumbar MRI, were thoroughly reviewed to assess the side of the lesions (unilateral or bilateral), migration of the herniated disc, broadly herniated discs, decreased intensity of the disc on T2-weighted MRI, as well as the presence of high-intensity zones (HIZs), thecal sac compression, spondylolisthesis, central stenosis, and foraminal stenosis. Decreased intensity of the disc on T2weighted MRI indicated a lack of homogeneity with a hypointense grey or black signal intensity and the loss of distinction between the nucleus and annulus ${ }^{29,30}$. Procedure-related variables, including the number of treatment levels and post-procedural complications over 6 months after the procedure, were also recorded.

\section{Definition of a successful outcome.}

The NRS pain and ODI scores before and at 1,2,3, and 6 months after the procedure were determined through chart reviews. Patients whose NRS pain scores decreased by $50 \%$ or more and whose ODI scores decreased by $40 \%$ or more at 6 months after the procedure than before the procedure were grouped into the successful outcome group. Patients who were lost during the 6-month follow-up $(n=4)$ were grouped into the unsuccessful outcome group. According to these definitions, 80 patients had successful outcomes and 26 had unsuccessful outcomes at 6 months after the procedure. 


\section{Statistical analysis.}

Normality of data was analysed using the Kolmogorov-Smirnov test for all continuous parameters. Continuous variables were presented as mean and standard deviation (SD) or median and interquartile range, depending on normality. Categorical demographic variables were reported as a number or a percentage. For continuous variables, comparisons of patient characteristics between the groups were made using Student's t-tests or the Mann-Whitney $U$ test. Categorical demographic data were analysed using Pearson's chi-square test or Fisher's exact test. P values less than 0.05 were considered statistically significant. To compare the NRS pain and ODI scores before and after the procedure, the Wilcoxon signed-rank test was performed. The P level was adjusted $(0.05 / 4=0.0125)$ for the multiple comparisons of the differences in the scores at 1,2,3, and 6 months versus those before the procedure. To quantify the relationship between successful outcomes and the patients' clinical and demographic characteristics, binary logistic regression techniques were used. To determine independent positive prognostic factors of the procedure, multivariable logistic regression analysis was performed using variables that were statistically significant in the univariable analysis. By implementing the Hosmer-Lemeshow goodnessof-fit test, the regression model was determined to be appropriate. All data were analysed using IBM SPSS Statistics for Windows/Macintosh, Version 24.0 (IBM Corp., Armonk, NY).

\section{Results}

\section{Demographics.}

Patient demographics and clinical characteristics are listed in Table 1. Of the 106 patients included, 74 $(69.8 \%)$ had unilaterally herniated discs. Migrations of the herniated discs were found in 18 patients (17.0\%). HIZs and thecal sac compression were found in 63 (59.4\%) and 69 patients (65.1\%), respectively. 
Table 1

Comparison of demographic and clinical variables between the successful and unsuccessful outcome groups according to the NRS pain and ODI scores.

\begin{tabular}{|c|c|c|c|c|}
\hline Variable & $\begin{array}{l}\text { Total }(n= \\
\text { 106) }\end{array}$ & $\begin{array}{l}\text { Successful (n } \\
=80)\end{array}$ & $\begin{array}{l}\text { Unsuccessful ( } \mathrm{n} \\
=26)\end{array}$ & $\begin{array}{l}P \\
\text { value }\end{array}$ \\
\hline Age (years), mean $\pm S D$ & $49.8 \pm 14.5$ & $48.7 \pm 12.8$ & $53.3 \pm 18.6$ & 0.244 \\
\hline Sex (male), N (\%) & $56(52.8)$ & $41(51.3)$ & $15(57.7)$ & 0.568 \\
\hline $\mathrm{BMI}\left(\mathrm{kg} / \mathrm{m}^{2}\right)$, mean $\pm \mathrm{SD}$ & $23.5 \pm 3.4$ & $23.7 \pm 3.5$ & $22.9 \pm 3.0$ & 0.248 \\
\hline $\begin{array}{l}\text { Duration of symptoms (months), } \\
\text { median (IQR) }\end{array}$ & $180(300)$ & $180(292.5)$ & $210(300)$ & 0.718 \\
\hline History of smoking, $\mathrm{N}(\%)$ & $16(15.1)$ & $9(11.3)$ & $7(26.9)$ & 0.064 \\
\hline \multicolumn{5}{|l|}{ Number of treatment levels, $\mathrm{N}(\%)$} \\
\hline 1 level & $78(73.6)$ & $55(68.8)$ & $23(88.5)$ & $0.048 *$ \\
\hline 2 levels or more & $28(26.4)$ & $25(51.3)$ & $3(11.5)$ & \\
\hline \multicolumn{5}{|l|}{ Side of lesion, N (\%) } \\
\hline Left or right side & $74(69.8)$ & $65(81.3)$ & $9(34.6)$ & $<.001 *$ \\
\hline Both sides & $32(30.2)$ & $15(18.8)$ & $17(65.4)$ & \\
\hline Migration of the herniated disc, $\mathrm{N}(\%)$ & $18(17.0)$ & $9(11.3)$ & $9(34.6)$ & $0.013^{*}$ \\
\hline Broadly herniated disc, N (\%) & $37(34.9)$ & $12(46.2)$ & $25(31.3)$ & 0.166 \\
\hline $\begin{array}{l}\text { Decreased intensity of the disc on } \\
\text { T2-WI, N (\%) }\end{array}$ & $97(91.5)$ & $74(92.5)$ & $23(88.5)$ & 0.686 \\
\hline High-intensity zone, N (\%) & $63(59.4)$ & $53(66.3)$ & $10(38.5)$ & $0.012^{\star}$ \\
\hline Thecal sac compression, $\mathrm{N}(\%)$ & $69(65.1)$ & $46(57.5)$ & $23(88.5)$ & $0.004 *$ \\
\hline Spondylolisthesis, N (\%) & $15(14.2)$ & $11(13.8)$ & $4(15.4)$ & 1.000 \\
\hline Central stenosis, N (\%) & $46(43.4)$ & $32(40.0)$ & $14(53.8)$ & 0.216 \\
\hline Foraminal stenosis, N (\%) & $77(72.6)$ & $55(68.8)$ & $22(84.6)$ & 0.115 \\
\hline History of surgery, N (\%) & $6(5.7)$ & $5(6.3 \%)$ & $1(3.9 \%)$ & 1.000 \\
\hline \multicolumn{5}{|c|}{$\begin{array}{l}\text { P values are obtained using Pearson's chi-square test or Fisher's exact test and Student's t-test or the } \\
\text { Mann-Whitney U test. *indicates a significant difference. NRS pain: Numeric rating scale for pain; } \\
\text { ODI: Oswestry disability index; SD: standard deviation; BMI: body mass index; IQR: interquartile range; } \\
\text { T2-WI: T2-weighted magnetic resonance image. }\end{array}$} \\
\hline
\end{tabular}




\section{Characteristics of the successful and unsuccessful outcome groups.}

Table 1 shows a comparison between the demographic and clinical variables among the patients in the successful versus unsuccessful outcome groups. Among the 106 patients, 80 (75.5\%) experienced a successful outcome after PDD using the L'DISQ (Fig. 1). The outcome was significantly better among patients who underwent the procedure at one vertebral level than among patients who underwent the procedure at two or more levels $(P=0.048)$. The successful outcome group also included more patients with unilateral disc lesions than those with bilateral lesions $(P<0.001)$. Migration of the herniated disc $(P$ $=0.013)$ and thecal sac compression $(P=0.012)$ were more frequent in the unsuccessful outcome group than in the successful outcome group. However, HIZs were more frequent in the successful outcome group than in the unsuccessful outcome group $(P=0.012)$.

\section{Clinical efficacy of PDD using the L'DISQ.}

Figure 2 and Table 2 show the changes over time in the NRS pain and ODI scores after PDD using the L'DISQ. The mean baseline NRS pain and ODI scores were not significantly different between the successful and unsuccessful outcome groups ( $P=0.391$ for NRS pain score; $P=0.151$ for ODI score). However, the mean NRS pain scores \pm SD were lower at 1 month after the procedure than before $(77.6 \pm$ 16.2 versus $40.8 \pm 23.7, P<0.001)$, and were even lower at 6 months after the procedure than before $(28.7 \pm 22.8, \mathrm{P}<0.001)$. The mean ODI scores \pm SD were also lower at 1 month after the procedure than before $(56.6 \pm 20.3$ versus $33.2 \pm 17.5, P<0.001)$, and were even lower at 6 months after the procedure than before $(24.4 \pm 16.4, \mathrm{P}<0.001)$.

Table 2

Comparison of the NRS pain and ODI scores before and after the procedure.

\begin{tabular}{|c|c|c|c|c|c|c|}
\hline & $\begin{array}{l}\text { Before the } \\
\text { procedure }(n=106)\end{array}$ & $\begin{array}{l}1 \text { month }(n \\
=106)\end{array}$ & $\begin{array}{l}2 \text { months } \\
(n=105)\end{array}$ & $\begin{array}{l}3 \text { months (n } \\
=105)\end{array}$ & $\begin{array}{l}6 \text { months (n } \\
=102)\end{array}$ & $\begin{array}{l}P \\
\text { value }\end{array}$ \\
\hline $\begin{array}{l}\text { NRS } \\
\text { pain }\end{array}$ & $77.6 \pm 16.2$ & $40.8 \pm 23.7$ & $31.1 \pm 21.1$ & $29.1 \pm 21.9$ & $28.7 \pm 22.8$ & $<$ \\
\hline ODI & $56.6 \pm 20.3$ & $33.2 \pm 17.5$ & $27.0 \pm 17.4$ & $24.7 \pm 15.6$ & $24.4 \pm 16.4$ & $<.001 *$ \\
\hline \multicolumn{7}{|c|}{$\begin{array}{l}\text { Values are the mean } \pm \text { standard deviation. Wilcoxon signed-rank test is used to compare the mean } \pm \\
\text { standard deviation. Bonferroni correction is used to adjust for the differences in values at } 1,2,3 \text {, and } \\
6 \text { months after the procedure versus those before the procedure. * indicates a significant difference. }\end{array}$} \\
\hline
\end{tabular}

\section{Factors associated with the prognosis after PDD using the L'DISQ.}


Table 3 shows the factors associated with the outcomes determined using both univariable and multivariable analyses. Demographic characteristics and variables showing a trend toward statistical significance in the univariable analyses, including bilaterally herniated discs $(P<0.001)$, migration of the herniated disc $(P=0.009), H I Z(P=0.014)$, and thecal sac compression $(P=0.008)$, were included in the multivariable logistic regression analyses. Among the selected clinical variables, the presence of bilaterally herniated discs (adjusted odds ratio $[\mathrm{aOR}]=6.757 ; \mathrm{P}<0.001$ ), migration of the herniated disc $(a O R=5.116 ; P=0.017)$, and absence of $\mathrm{HIZs}(a O R=0.254 ; \mathrm{P}=0.016)$ were negative predictors of successful PDD using the L'DISQ. 
Table 3

Factors associated with unsuccessful outcomes after PDD using L'DISQ.

\begin{tabular}{|c|c|c|c|c|}
\hline \multirow[t]{2}{*}{ Variable } & \multicolumn{2}{|c|}{ Univariable analysis } & \multicolumn{2}{|c|}{$\begin{array}{l}\text { Multivariable analysis }\left(\mathrm{R}_{\mathrm{N}}{ }^{2}=\right. \\
0.407)\end{array}$} \\
\hline & OR (95\% Cl) & $P$ & $\begin{array}{l}\text { Adjusted OR (95\% } \\
\mathrm{Cl})\end{array}$ & $\begin{array}{l}P \\
\text { value }\end{array}$ \\
\hline Age & $\begin{array}{l}1.023(0.991- \\
1.056)\end{array}$ & 0.158 & & \\
\hline Female sex & $\begin{array}{l}0.771(0.316- \\
1.883)\end{array}$ & 0.568 & & \\
\hline BMI & $\begin{array}{l}0.922(0.804- \\
1.058)\end{array}$ & 0.247 & & \\
\hline Duration of symptoms & $\begin{array}{l}0.999(0.998- \\
1.001)\end{array}$ & 0.350 & & \\
\hline History of smoking & $\begin{array}{l}2.906(0.958- \\
8.818)\end{array}$ & 0.060 & & \\
\hline Procedure at 2 or more levels & $\begin{array}{l}0.287(0.079- \\
1.045)\end{array}$ & 0.058 & & \\
\hline Both sides & $\begin{array}{l}8.185(3.060- \\
21.894)\end{array}$ & $<.001^{*}$ & $\begin{array}{l}6.757(2.233- \\
20.446)\end{array}$ & $0.001 *$ \\
\hline Migration of the herniated disc & $\begin{array}{l}4.176(1.440- \\
12.112)\end{array}$ & $0.009 *$ & $\begin{array}{l}5.116(1.337- \\
19.576)\end{array}$ & $0.017 *$ \\
\hline Broadly herniated disc & $\begin{array}{l}1.886(0.763- \\
4.659)\end{array}$ & 0.169 & & \\
\hline $\begin{array}{l}\text { Decreased intensity of the disc on } \\
\text { T2-WI }\end{array}$ & $\begin{array}{l}0.622(0.144- \\
2.684)\end{array}$ & 0.524 & & \\
\hline High-intensity zone & $\begin{array}{l}0.318(0.127- \\
0.796)\end{array}$ & $0.014^{\star}$ & $0.254(0.083-0.774)$ & $0.016^{*}$ \\
\hline Thecal sac compression & $\begin{array}{l}5.667(1.572- \\
20.426)\end{array}$ & $0.008^{*}$ & $\begin{array}{l}3.017(0.723- \\
12.595)\end{array}$ & 0.130 \\
\hline Spondylolisthesis & $\begin{array}{l}1.140(0.330- \\
3.945)\end{array}$ & 0.836 & & \\
\hline Central stenosis & $\begin{array}{l}1.750(0.718- \\
4.267)\end{array}$ & 0.219 & & \\
\hline Foraminal stenosis & $\begin{array}{l}2.500(0.779- \\
8.019)\end{array}$ & 0.123 & & \\
\hline
\end{tabular}

* indicates a significant difference. OR: odds ratio; Cl: confidence interval; BMl: body mass index; T2WI: T2-weighted magnetic resonance image. 


\begin{tabular}{|c|c|c|c|c|}
\hline \multirow[t]{2}{*}{ Variable } & \multicolumn{2}{|c|}{ Univariable analysis } & \multicolumn{2}{|c|}{$\begin{array}{l}\text { Multivariable analysis }\left(\mathrm{R}_{\mathrm{N}}{ }^{2}=\right. \\
0.407)\end{array}$} \\
\hline & OR $(95 \% \mathrm{Cl})$ & $\begin{array}{l}\mathrm{P} \\
\text { value }\end{array}$ & $\begin{array}{l}\text { Adjusted OR (95\% } \\
\text { Cl) }\end{array}$ & $\begin{array}{l}P \\
\text { value }\end{array}$ \\
\hline History of surgery & $\begin{array}{l}0.600(0.067- \\
5.384)\end{array}$ & 0.648 & & \\
\hline
\end{tabular}

\section{Complications.}

Two cases of adverse events were observed in which pain did not improve after the procedure. In one case, the patient subjectively complained of mild weakness immediately after the procedure, but no abnormalities were noted on a neurologic examination. After a few hours of observation in the recovery room, these symptoms disappeared and the patient was discharged. In another case, cauda equina syndrome occurred after 1 month, regardless of the procedure. This patient opted to undergo spine surgery. Notably, only 4 of the 106 patients who underwent PDD using the L'DISQ received subsequent spine surgery because of insufficient reduction in their pain intensity. No other severe complications associated with the procedure were noted during follow-up.

\section{Discussion}

To the best of our knowledge, this is the first study to propose the prognostic predictors for successful PDD using the L'DISQ for the treatment of lumbar discogenic pain. The presence of HIZs, absence of migration of the herniated disc, and presence of unilaterally herniated discs were positive prognostic factors. A HIZ is a high-intensity signal on T2-weighted MRI located in the annulus fibrosus and is distinct from that in the nucleus pulposus. Aprill and Bogduk ${ }^{31}$ reported that HIZs were observed in $28 \%$ of patients with symptoms. HIZs have clinical significance as highly specific markers for painful lumbar discs; nevertheless, this notion has been controversial because HIZs are also found in patients without symptoms. In a symptomatic patient with a HIZ and without other causes of lumbar discogenic pain, the $\mathrm{HIZ}$ could indicate the problematic disc that causes pain ${ }^{32}$. In our study protocol, the target levels were determined on the basis of a combination of the patients' symptoms, physical examination findings, presence of HIZs on lumbar MRI, and response to prior diagnostic epidural blocks. Provocative discography was not performed because previous reports have proved that it could cause disc tissue damage and exacerbate disc degeneration ${ }^{33,34}$. Therefore, detecting the presence of HIZs as a definite sign of a pathologic lesion might contribute to successful treatment outcomes because it could help lower the likelihood of overlooking the lesion and delaying treatment. 
Migration of the herniated disc may also contribute to lowering the rate of successful outcomes. We postulated that advancing the tip of the wand to the migrated part of the disc may be technically difficult in such cases. Additionally, if the patient has bilaterally herniated discs, the target treatment area will be wider than that in patients with unilaterally herniated discs. Insufficient lesioning of the disc would also make it difficult to obtain a successful outcome. Minimally invasive disc decompression could be less effective in reducing pain because of insufficient removal of disc tissue in patients with large disc herniation, disc extrusion, or disc sequestration. This could also be the reason why Sharps and Issac ${ }^{24}$ set herniation greater than one-third of the sagittal diameter of the spinal canal, disc extrusion, or disc sequestration as a contraindication to nucleoplasty.

Among the various minimally invasive decompression techniques previously implemented to mitigate lumbar discogenic pain, the current technique differs from nucleoplasty, which also uses the coblation technology, because the protruded disc can be removed by directly accessing it using a navigable wand. To date, no study has directly compared nucleoplasty and PDD using the L'DISQ. However, on reviewing studies that reported the effects of nucleoplasty, we noted that Sharps and Isaac's study ${ }^{24}$ showed a success rate of $79 \%$ based on a 2-point reduction in the pain score at the 1-year follow-up after the procedure. In Singh et al.'s study ${ }^{26}$, when the pain score decreased by 2 points or more, the success rates were only $56 \%$ after 6 months and $59 \%$ after 1 year of the procedure. Although we defined a successful outcome as a $50 \%$ or greater reduction in the NRS pain scores and a $40 \%$ or greater reduction in the ODI scores after the procedure than before, our success rate was still higher than that of nucleoplasty. We attribute this better clinical effect to the lesioning in the target disc, which was possible via direct access afforded by the navigable wand, rather than a mere reduction in disc pressure.

Our study differs from existing ones on L'DISQ27,28 in that we analysed a much higher number of patients and obtained a different success rate. Lee et al. ${ }^{27}$ reported a success rate of $88 \%$ after 6 months of PDD using the L'DISQ, but their criterion of a successful outcome was based only on a greater than $50 \%$ decrease in the pain score. In contrast, we considered not only the pain score but also the functional score in defining a successful outcome; these stricter criteria used for defining a successful outcome could explain the difference in findings. In addition, Lee et al. ${ }^{27}$ limited the procedure to a single vertebral level, whereas we also analysed patients with lesioned discs at two or more levels. This could be an additional factor that explains the difference in the success rates.

Since L'DISQ ablates tissues using a bipolar radiofrequency energy, it poses a risk of damaging normal tissues owing to high temperatures, but a previous study using cadavers has demonstrated the temperature-related safety of this technique ${ }^{35}$. The temperature in the centre of the nucleus pulposus did not increase by more than $13^{\circ} \mathrm{C}$, and no pathological changes occurred in the surrounding tissues. Nevertheless, buttock or leg pain should be thoroughly monitored during the procedure to avoid any complications. Moreover, if muscle contraction in the lower extremity occurs because of electrical stimulation, the wand tip should be repositioned. In our study, no cases of serious complications were noted because of the careful attention paid during the procedure. 
This study has a few limitations. First, owing to the retrospective design, we could not include a control group. It would not be ethically possible to use a sham procedure. Further clinical trials are warranted to ascertain additional objective data about the efficacy of the L'DISQ compared to other procedures. Second, because the characteristics of the disc on MRI were used as a predictor of the procedure, predicting the effect when two or more levels are involved becomes more complicated.

\section{Conclusion}

In this study, PDD using the L'DISQ was found to be a safe and effective option for treating lumbar discogenic pain. Approximately $75.5 \%$ of the included patients showed a $50 \%$ or greater pain reduction and at least a $40 \%$ functional improvement without any severe complications. The presence of HIZs, absence of migration of the herniated disc, and presence of unilaterally herniated discs could be considered positive predictors for a successful outcome after PDD using the L'DISQ. We hope that the clinical implication of these results will be helpful for improving the efficacy of PDD.

\section{Declarations}

\section{Data availability}

The datasets obtained in the current study are available from the corresponding author on reasonable request.

\section{Acknowledgements}

The corresponding author would like to thank Prof. Jong Min Park who encouraged him to join this field of research.

\section{Author contributions}

J.Y.K., K.S.L., and S.M.J..: Collected and analysed the data, and drafted the manuscript. Y.H.K.: Critically reviewed the manuscript for important intellectual content. Y.H.K.: Designed the whole study, analysed and interpreted the data, and critically reviewed the manuscript for important intellectual content. J.Y.K. and Y.H.K.: Designed the whole study, and analysed and interpreted the data. All authors reviewed the manuscript.

\section{Additional information}

Correspondence and requests for materials should be addressed to Y.H.K. 


\section{Funding}

None

\section{Competing interests}

The authors declare no competing interests.

\section{References}

1. Schwarzer, A. C. et al. The Prevalence and Clinical Features of Internal Disc Disruption in Patients With Chronic Low Back Pain. Spine (Phila Pa 1976), 20, 1878-1883 (1995).

2. Moneta, G. B. et al. Reported Pain During Lumbar Discography As a Function of Anular Ruptures and Disc Degeneration: A Re-analysis of 833 Discograms. Spine (Phila Pa 1976), 19, 1968-1974 (1994).

3. Kloth, D. S., Fenton, D. S., Andersson, G. B. \& Block, J. E. Intradiscal electrothermal therapy (IDET) for the treatment of discogenic low back pain: patient selection and indications for use., 11, 659-668 (2008).

4. Laxmaiah, M., Vijay, S., Vidyasagar, P., Frank, J. E. F., Joshua, A. H. \& and Comparison of the Efficacy of Caudal, Interlaminar, and Transforaminal Epidural Injections in Managing Lumbar Disc Herniation: Is One Method Superior to the Other? Korean J. Pain, 28, 11-21 (2015).

5. Peng, B. et al. Possible Pathogenesis of Painful Intervertebral Disc Degeneration. Spine (Phila Pa 1976), 31, 560-566 (2006).

6. Freemont, A. J. et al. Nerve ingrowth into diseased intervertebral disc in chronic back pain. The Lancet, 350, 178-181 (1997).

7. Ruchi, G., Saru, S., Sukhdeep, K., Kulvinder, S., Kuljeet, A. \& and Correlation between Epidurographic Contrast Flow Patterns and Clinical Effectiveness in Chronic Lumbar Discogenic Radicular Pain Treated with Epidural Steroid Injections Via Different Approaches. Korean J. Pain, 27, 353-359 (2014).

8. Ahn, Y. \& Lee, S. H. Outcome predictors of percutaneous endoscopic lumbar discectomy and thermal annuloplasty for discogenic low back pain. Acta Neurochir. (Wien), 152, 1695-1702 (2010).

9. Andersson, G. B., Mekhail, N. A. \& Block, J. E. Treatment of intractable discogenic low back pain. A systematic review of spinal fusion and intradiscal electrothermal therapy (IDET)., 9, 237-248 (2006).

10. Vamvanij, V., Fredrickson, B. E., Thorpe, J. M., Stadnick, M. E. \& Yuan, H. A. Surgical treatment of internal disc disruption: an outcome study of four fusion techniques. J. Spinal Disord, 11, 375-382 (1998).

11. Laxmaiah, M., Peter, S. S., Devi, E. N., Joshua, A. H. \& and What is the Role of Epidural Injections in the Treatment of Lumbar Discogenic Pain: A Systematic Review of Comparative Analysis with Fusion. Korean J. Pain, 28, 75-87 (2015). 
12. Archavlis, E. \& Carvi, M. Comparison of minimally invasive fusion and instrumentation versus open surgery for severe stenotic spondylolisthesis with high-grade facet joint osteoarthritis. Eur. Spin J. 22, 1731-1740(2013).

13. Bono, C. M. et al. Pain intensity and patients' acceptance of surgical complication risks with lumbar fusion. Spine (Phila Pa 1976), 38, 140-147 (2013).

14. Manchikanti, L. et al. An update of the systematic assessment of mechanical lumbar disc decompression with nucleoplasty., 16, Se25-54 (2013).

15. Manchikanti, L. et al. Evaluation of the relative contributions of various structures in chronic low back pain., 4, 308-316 (2001).

16. Freeman, B. J. C. et al. Double-Blind, Controlled Trial: Intradiscal Electrothermal Therapy: Versus: Placebo for the Treatment of Chronic Discogenic Low Back Pain. Spine (Phila Pa 1976), 30, 23692377 (2005).

17. Chen, Y., Derby, R. \& Lee, S. H. Percutaneous disc decompression in the management of chronic low back pain. Orthop. Clin. North Am, 35, 17-23 (2004).

18. Pauza, K. J. et al. A randomized, placebo-controlled trial of intradiscal electrothermal therapy for the treatment of discogenic low back pain. Spine J, 4, 27-35 (2004).

19. Finch, P. M., Price, L. M. \& Drummond, P. D. Radiofrequency heating of painful annular disruptions: one-year outcomes. J. Spinal Disord. Tech, 18, 6-13 (2005).

20. Klein, R. G. et al. Biochemical injection treatment for discogenic low back pain: a pilot study. Spine J, 3, 220-226 (2003).

21. Derya Burcu, H., Arsal, A., Eric, H. \& and R. The outcome of epiduroscopy treatment in patients with chronic low back pain and radicular pain, operated or non-operated for lumbar disc herniation: a retrospective study in 88 patients. Korean J. Pain, 31, 109-115 (2018).

22. Eun Hee, C., Soo, H. \& and P. A Modified Approach of Percutaneous Endoscopic Lumbar Discectomy (PELD) for Far Lateral Disc Herniation at L5-S1 with Foot Drop. Korean J. Pain, 29, 57-61 (2016).

23. Sei, F. et al. Results of Intradiscal Pulsed Radiofrequency for Lumbar Discogenic Pain: Comparison with Intradiscal Electrothermal Therapy. Korean J. Pain, 25, 155-160 (2012).

24. Sharps, L. S. \& Isaac, Z. Percutaneous disc decompression using nucleoplasty., 5, 121-126 (2002).

25. Chen, Y. C., Lee, S. H. \& Chen, D. Intradiscal pressure study of percutaneous disc decompression with nucleoplasty in human cadavers. Spine (Phila Pa 1976), 28, 661-665 (2003).

26. Singh, V., Piryani, C., Liao, K. \& Nieschulz, S. Percutaneous disc decompression using coblation (nucleoplasty) in the treatment of chronic discogenic pain., 5, 250-259 (2002).

27. Lee, S. H. et al. Efficacy of a new navigable percutaneous disc decompression device (L'DISQ) in patients with herniated nucleus pulposus related to radicular pain. Pain Med, 12, 370-376 (2011).

28. Lee, S. H. et al. Effectiveness of a new navigable percutaneous disc decompression device (L'DISQ) in patients with lumbar discogenic pain. Pain Med, 16, 266-273 (2015). 
29. Pfirrmann, C. W., Metzdorf, A., Zanetti, M., Hodler, J. \& Boos, N. Magnetic resonance classification of lumbar intervertebral disc degeneration. Spine (Phila Pa 1976), 26, 1873-1878 (2001).

30. Riesenburger, R. I., Safain, M. G., Ogbuji, R., Hayes, J. \& Hwang, S. W. A novel classification system of lumbar disc degeneration. J. Clin. Neurosci, 22, 346-351 (2015).

31. Aprill, C. \& Bogduk, N. High-intensity zone: a diagnostic sign of painful lumbar disc on magnetic resonance imaging. Br. J. Radiol, 65, 361-369 (1992).

32. Khan, I., Hargunani, R. \& Saifuddin, A. The lumbar high-intensity zone: 20 years on. Clin. Radiol, 69, 551-558 (2014).

33. Provenzano, D. A. Diagnostic discography: what is the clinical utility? Curr. Pain Headache Rep, 16, 26-34 (2012).

34. Zhang, Y. G., Guo, T. M., Guo, X. \& Wu, S. X. Clinical diagnosis for discogenic low back pain. Int. J. Biol. Sci, 5, 647-658 (2009).

35. Hong, Y. et al. Feasibility of Navigable Percutaneous Disk Decompressor (L'DISQ-C) for Cervical Disk Herniation. J. Neurol. Surg. A. Cent. Eur. Neurosurg, 79, 231-238 (2018).

\section{Figures}

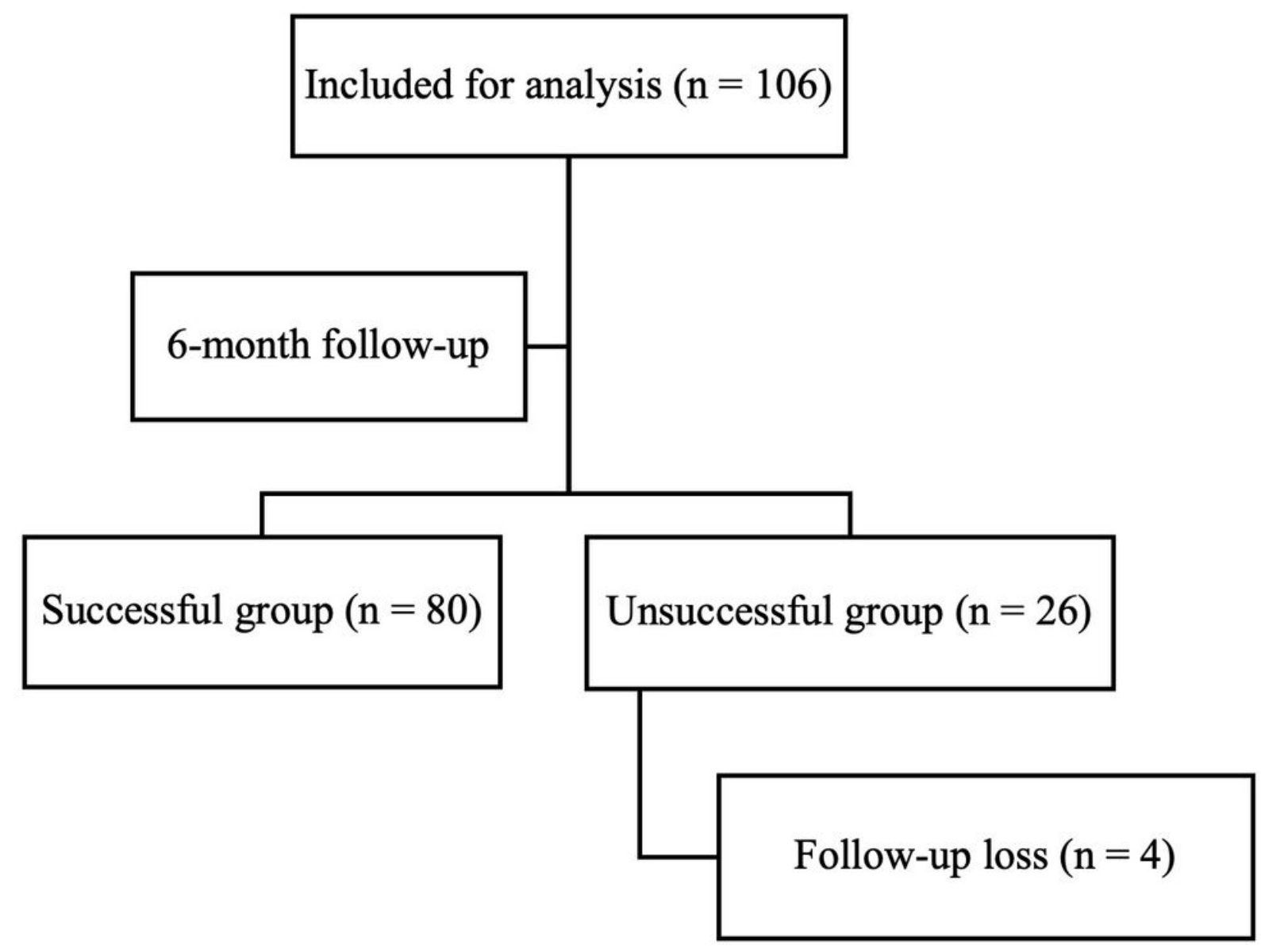

Figure 1 
Schematic diagram of the study.

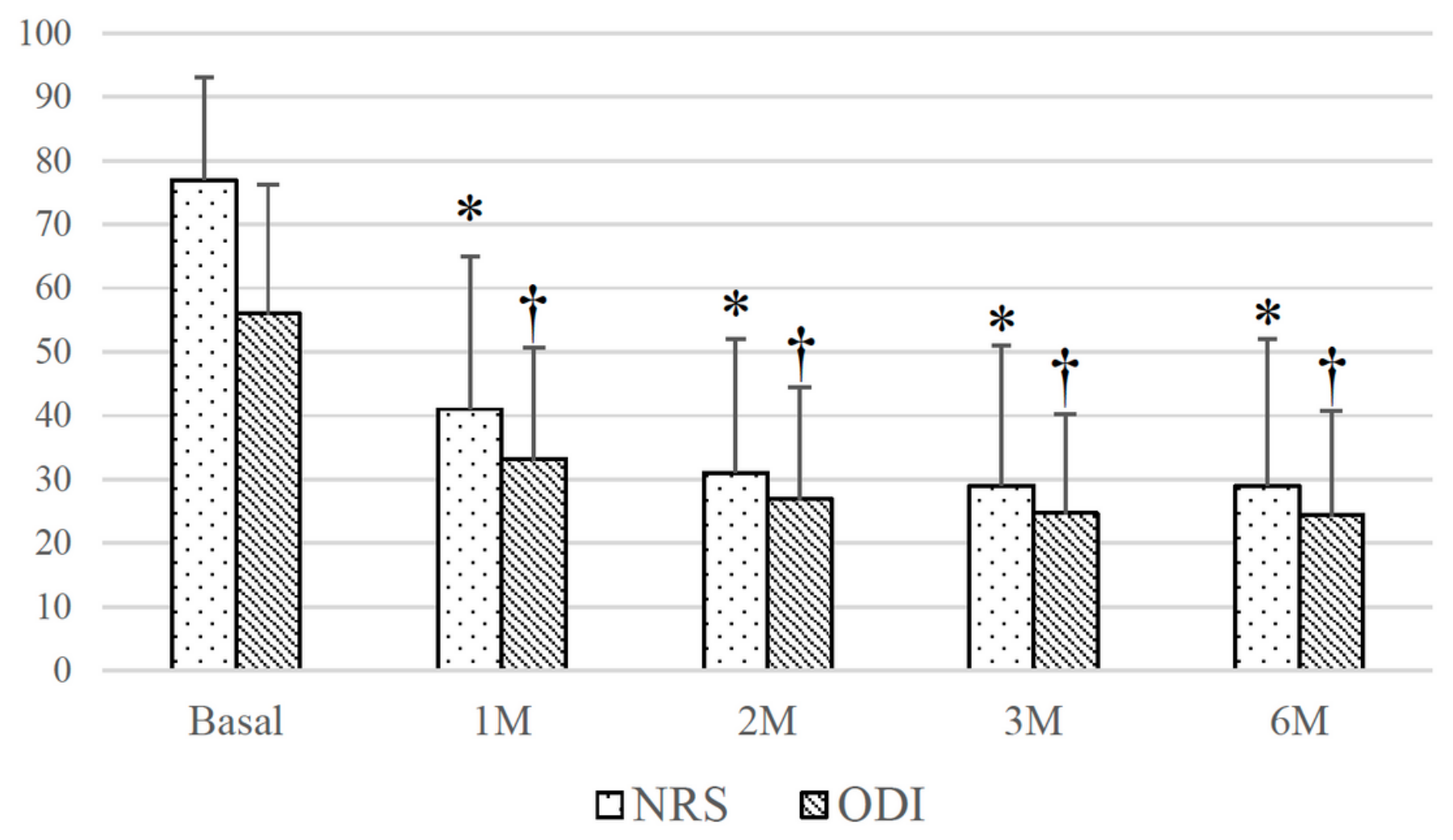

Figure 2

Changes in the NRS pain and ODI scores during the follow-up period. * and + indicate significant differences compared with the baseline $(P<0.0125$; Bonferroni correction and Wilcoxon signed-rank test). NRS pain: Numeric rating scale for pain; ODI: Oswestry disability index. 